\title{
A comparison of the frequency of major affective disorder in Huntington's disease and Alzheimer's disease
}

\author{
RHS MINDHAM, ${ }^{*}$ CYNTHIA STEELE, $\uparrow$ MF FOLSTEIN, $\dagger$ JANE LUCAS $\dagger$ \\ From the University Department of Psychiatry, Leeds, ${ }^{*} U K$, and the Johns Hopkins Hospital, Baltimore, $\dagger$ \\ USA
}

SUMMARY Matched groups of patients suffering from Huntington's disease and Alzheimer's disease were compared for psychiatric morbidity prior to the onset of dementia. The Huntington's disease group showed twice the incidence of major affective disorder. This finding suggests a specific relationship between Huntington's disease and major affective disorder rather than the latter being a non-specific prodromal feature of dementia.

Dementia is a major feature of Huntington's disease and is eventually seen in most sufferers from the condition. In addition to dementia, many psychiatric syndromes have been described in patients with the disease but it is unclear whether these illnesses should be regarded as coincidental, as reactions to Huntington's disease and its effects, or as illnesses intimately linked with Huntington's disease. In this context, the greatest interest has centred on the association between Huntington's disease and major affective disorder.'

A recent review has drawn attention to the lack of agreement as to the nature and origins of affective symptoms in patients suffering from Huntington's disease. $^{2}$ On the one hand the symptoms are seen as manifestations of a major affective disorder indistinguishable from that occurring in patients not suffering from organic brain disease; and on the other, the symptoms are seen as secondary to the changes in the brain in Huntington's disease and different from major affective disorder in clinical presentation. There are important problems in relating the symptomatology observed to that seen in other disorders. The classification of psychiatric disorders has been greatly influenced by hierarchical concepts from Kraepelin's time and these are explicitly stated

\footnotetext{
Address for reprint requests: Professor RHS Mindham, Department of Psychiatry. University of Leeds, 15 Hyde Terrace, Leeds LS2 9LT, UK.
}

Received 21 January 1985 and in revised form 15 March 1985. Accepted 23 March 1985 in DSM III. ${ }^{3}$ In evaluating causative factors for symptoms, organic conditions of the brain take precedence over all other conditions making it difficult conceptually to assess the likely coincidence of two conditions from different levels in the hierarchy. The reviewers believe that this had greatly influenced German thinking on the relationship between Huntington's disease and affective symptoms. Another important factor is the broadness or narrowness of concept of affective illness applied.

We are particularly concerned with the relationship between affective symptoms, and organic brain disease in Huntington's disease. As dementia is the psychiatric syndrome most consistently found in Huntington's disease it might be postulated that manic-depressive illness prior to the onset of Huntington's disease is a prodromal feature of dementia. If the occurrence of major affective disorder in Huntington's disease is simply an early manifestation of dementia, a similarly frequent occurrence of major affective disorder might be expected as a prodromal feature of other dementing illnesses. To examine the relationship between these psychiatric syndromes in Huntington's disease we decided to assess the frequency of major affective disorder in demented subjects prior to the onset of dementia, and to compare the occurrence of the disorder in sufferers from Huntington's disease and Alzheimer's disease. To facilitate this work, we adopted the following hypothesis: "The major affective disorders occasionally reported prior to the onset of dementia occur with similar frequency whatever the pathological nature of the dementing illness". 
Method

A series of patients suffering from Alzheimer's disease was randomly selected from an outpatient clinic specialising in the diagnosis and management of the disorder. The patients suffering from Alzheimer's disease were matched by sex with patients suffering from Huntington's disease with dementia; these subjects were taken from the register of the Baltimore Huntington's Disease Project which covers the whole of Maryland. ${ }^{4}$ In each case only the oldest affected member of the family was taken. This was done to make the ages of the two groups as similar as possible and to avoid the inclusion of several members of a Huntington's disease family in which major affective disorder might be particularly common.

A diagnosis of Huntington's disease was made on the presence of choreiform movements, delayed in onset but progressing in severity; associated with a family history of a similar illness compatible with autosomal dominant inheritance. ${ }^{5}$

A diagnosis of Alzheimer's disease was made where a dementia syndrome as defined in the Third Edition of the Diagnostic and Statistical Manual of Mental Disorders (DSM III) of the American Psychiatric Association was of sufficient severity to interfere with social or occupational functioning and in the absence of evidence of: a history of strokes or transient ischaemic attacks; diabetes mellitus; hypertension requiring treatment; alcoholism; head injuries associated with loss of consciousness; brain tumour; epilepsy; Parkinsonism, or any other indication of specific disease of the central nervous system. ${ }^{6}$ A history of psychiatric illness did not exclude a diagnosis of Alzheimer's disease.

Information concerning the patients in both groups was collected using the National Institute of Mental Heaith Diagnostic Interview Schedule (DIS). In view of the evidence of dementia in all patients studied, information from relatives was invariably obtained and particular care was taken in eliciting details of previous psychiatric illness. Information on the two groups was collected separately so that the interviewers did not know the psychiatric characteristics of the other group. The information collected was used to allocate a psychiatric diagnosis to each patient according to the categories of DSM III. Functional disorders were not diagnosed after the emergence of evidence of dementia; in these circumstances the organic illness was regarded as the primary disorder.

\section{Results}

Twenty-seven white patients (12 male, 15 female) from each of the two diagnostic groups were studied. The average age of the Alzheimer's disease patients (70.5 years) was greater than that of the Huntington's disease group (63.8 years): this difference was unavoidable in view of the great difference in the average age of onset of the two disorders. The difference in average ages of the groups means that the Alzheimer's group was exposed to the risk of psychiatric illness for a greater period of time than the Huntington's disease group.

Psychiatric illnesses suffered before the onset of dementia are shown in the table. Although the differences between the groups just fails to reach the normal criteria for statistical significance, it is evident that the psychiatric morbidity, in the course of the lifetime of subjects suffering from Huntington's disease, is greater than that among those with Alzheimer's disease $\left(\chi^{2}=3.68,1 \mathrm{df}, \mathrm{p}=0.06\right)$, in spite of the latter group's greater average age. Particularly striking is the frequency of major affective disorder in the Huntington's disease group, being twice that of the Alzheimer group.

\section{Discussion}

The findings of the study require the rejection of our initial hypothesis; the occurrence of major affective disorder in Huntington's disease is not of similar frequency in dementia of different pathological types and cannot be regarded as simply coincidental or as an early and non-specific sign of the dementing process. Our study does not give direct evidence of the reasons for the excessive occurrence of major affective disorder in the Huntington's disease group but the findings are compatible with a number of explanations, including the possibility of some kind of linked inheritance.

Our study has many methodological limitations:

Table Psychiatric illness suffered before the onset of dementia

\begin{tabular}{ll}
\hline Alzheimer's disease & Huntington's disease \\
\hline 296.20 & $296.20 ; 312.34$ \\
296.20 & 296.30 \\
296.20 & 296.30 \\
296.20 & 296.30 \\
300.40 & 296.30 \\
296.20 & $296.30 ; 301.70 ; 303.90$ \\
296.20 & $305.00 ; 312.34$ \\
303.90 & 296.30 \\
& $300.20 ; 312.34$ \\
& 312.34 \\
& 296.30 \\
& $296.60 ; 305.00$ \\
& 296.30 \\
& $296.30 ; 312.34$ \\
& 296.20 \\
\end{tabular}

Key to Table

Diagnostic Categories from DSM III

296.20 Major depression, single episode

296.30 Major depression, recurrent

296.40 Bipolar disorder, manic

296.50 Bipoiar disorder, depressed

296.60 Bipolar disorder, mixed

300.20 Phobic disorder

300.40 Dysthymic disorder

303.90 Alcohol dependence

301.70 Anti-social personality disorder

312.30 Anxiety disorder of childhood

305.00 Substance abuse 
evidence for psychiatric illness was collected retrospectively; the groups were not accurately matched for age; and the numbers studied were relatively small. On the other hand, the cases were well documented and were fully investigated once Huntington's disease or Alzheimer's disease had been suspected; all the cases were seen by the team and relatives were interviewed; and the occurrence of mental illness in the groups was high.

Although we have not resolved the many difficulties in satisfactorily defining and identifying affective illness, we have applied the same diagnostic criteria to each group. The diagnosis of non-organic illness was made with a knowledge of the nature of the dementing illness in each patient, but the workers concerned were unaware of the variety and frequency of psychiatric illness being identified in the two groups and were required to make the judgement on each case independently.

Our findings give support to the previously reported impressions that patients with Huntington's disease suffer from major affective disorder more often than would be expected by chance, or as a prelude to dementing illness. The relationship between Huntington's disease and major affective disorder deserves further close scrutiny; clarification would be useful in the diagnosis, management and counselling of patients and their families, and further light might be cast on the relationship between organic and affective disorders more widely.

RHS Mindham was supported by the University of Leeds during sabbatical leave in the USA; we received help from Barbara Jensen in the handling of data and from Hilary Cole in preparing this report.

\section{References}

' Folstein S, Abbott M, Folstein MF. Huntington's disease: association with major affective disorder.J Hum Gen 1981;33:76A.

${ }^{2}$ Koehler K, Sass H. "Affective psychopathology in Huntington's disease: the Johns Hopkins hypothesis and German psychiatry". Psychol Med 1984;14:733-7.

${ }^{3}$ American Psychiatric Association. Diagnostic and Statistical Manual of Mental Disorders (Third Edition) DSM III. Washington DC: The American Psychiatric Association, 1980.

${ }^{4}$ Folstein S, Abbott M, Chase GA, Jensen BA, Folstein MF. The association of affective disorder with Huntington's Disease in a case series and in families. Psycholog Med 1983b;13:537-42.

${ }^{5}$ Folstein S, Franz ML, Jensen B, Chase GA, Folstein MF. Conduct disorder and affective disorder among the offspring of patients with Huntington's disease. Psychol Med 1983a; 13:45-52.

${ }^{6}$ Breitner JCS, Folstein MF. Familial Alzheimer dementia: a prevalent disorder with specific features. Psycholog Med 1984;14:63-80. 